\title{
Estudo artroscópico da articulação do ombro em cadáveres de cães ${ }^{1}$
}

\author{
Arthroscopic study of the shoulder joint on dogs cadavers
}

\author{
Angélica Cecília Tatarunas ${ }^{2}$, Julia Maria Matera ${ }^{3}$ \\ 1. Trabalho realizado no Departamento de Cirurgia da Faculdade de Medicina Veterinária e Zootecnia \\ (FMVZ) da Universidade de São Paulo (USP). \\ 2. Pós-graduanda do Departamento de Cirurgia da FMVZ / USP. \\ 3. Professora Titular do Departamento de Cirurgia da FMVZ / USP.
}

\section{RESUMO}

OBJETIVO: Avaliar as dificuldades técnicas e possibilidade de visibilização das estruturas intra-articulares na artroscopia da articulação do ombro em cadáveres de cães.

MÉTODOS: Artroscopia foi realizada em 30 articulações utilizando-se os portais lateral para o artroscópio e craniolateral para o instrumental.

RESULTADOS: Lesões encontradas durante o estudo artroscópico consistiram de osteocondrose da cabeça umeral, presença de material estranho aderido à cápsula articular e lesão do ligamento glenoumeral medial, além daquelas da cartilagem promovidas de forma iatrogênica. Complicações decorrentes do procedimento consistiram de dificuldade na confecção dos portais artroscópico e instrumental e realização da técnica de triangulação, com deslocamento prematuro do artroscópio e instrumental e infiltração periarticular.

CONCLUSÃO: A utilização da artroscopia no cão é uma técnica difícil e requer prévio treinamento em cadáveres anterior à sua realização em casos clínicos.

Descritores: Artroscopia. Ombro. Cão. Articulação. Cadáver.

\section{ABSTRACT}

PURPOSE: The aim of this research was to analyze the technical difficulties and the possibility to see the structures into the joint on the arthroscopy of the shoulder on dogs cadavers.

METHODS: The portals were lateral for the arthroscopy and craniolateral for the instrument on the 30 joints studied.

RESULTS: On studying it the diagnosis showed osteocondrosis of the humeral head, strange material attached to the caudomedial gutter, tear of the glemoumeral medial ligament, and iatrogenic lesions of the cartilage. The complications concerning the technique consisted of the difficulty to do the portal arthroscopic, portal instrumental and triangulation. There was premature removal of both the arthroscopic and instrument as well as periarticular infiltration.

CONCLUSION: The use of the arthroscopy in dogs is a difficult technique and requires an early cadaver training before doing it in clinical cases. 
Key words: Arthroscopy. Shoulder. Dog. Joint. Cadáver.

\section{Introdução}

A artroscopia da articulação do ombro em cães foi descrita pela primeira vez por Person ${ }^{1}$ em 1986.

A principal indicação da técnica na espécie canina é o diagnóstico e tratamento de osteocondrite dissecante $^{2-5}$, mas também afecções do tendão do músculo bíceps braquial ${ }^{6}$, instabilidade $\operatorname{articular}^{7,8}$ e fraturas articulares ${ }^{7}$.

Diferentes portais foram relatados para o artroscópio e instrumentos nesta articulação. Inicialmente foram descritos 3 portais: cranial a cabeça acromial do Mm. deltóide e dorsal ao tubérculo maior, sobre a proeminência intertubercular ao nível do ligamento umeral transverso e 1 a $2 \mathrm{~cm}$ caudal e 1 a $2 \mathrm{~cm}$ distal ao acrômio ${ }^{1}$. Posteriormente Goring e Price ${ }^{9}$ utilizaram um portal artroscópico entre as duas cabeças do Mm deltóide, cerca de $2 \mathrm{~cm}$ distal ao acrômio. Rochat ${ }^{10}$ citou um portal artroscópio $1 \mathrm{~cm}$ distal ao acrômio e imediatamente caudal a cabeça acromial do Mm deltóide e Martini et al. ${ }^{11}$, a fim de simplificar as técnicas descritas anteriormente sugeriram a criação de dois portais, um cranial ao ligamento glenoumeral e outro caudal ( 1 a $2 \mathrm{~cm}$ distal e 3 a $4 \mathrm{~cm}$ caudal ao acrômio), os quais foram invertidos em sua função de portal telescópico ou instrumental de acordo com a região a ser tratada.

Falhas técnicas durante o estudo endoscópico da articulação do ombro compreendem inabilidade em criar um portal artroscópico ou instrumental adequados, explorar adequadamente a articulação, deslocamento prematuro do artroscópio, dificuldade de triangulação e lesão iatrogênica de cartilagem ${ }^{1,12,13}$, laceração do tendão do Mm. bíceps braquial e do tendão do Mm. Infraespinhoso ${ }^{1}$. 
A infiltração dos tecidos moles periarticulares, que é uma complicação que ocorre com relativa freqüência, resulta em distorção dos planos teciduais, perda das referências anatômicas e colapso da cápsula articular $7,10,11,14,15$.

O objetivo deste trabalho foi avaliar as dificuldades técnicas e possibilidades de visibilização das estruturas intra-articulares durante artroscopia da articulação do ombro em cadáveres de cães.

\section{Métodos}

Utilizou-se 15 cadáveres (30 articulações) de cães que vieram a óbito por diferentes motivos no Hospital Veterinário da Faculdade de Medicina Veterinária e Zootecnia da USP. Avaliou-se as dificuldades inerentes da técnica e a possibilidade de visibilização das estruturas intra-articulares, bem como a presença de alterações já existentes ou promovidas de forma iatrogênica. Após a artroscopia realizou-se estudo necroscópico confirmativo.

O material consistiu de telescópio ${ }^{1} 2.7 \mathrm{~mm}$, comprimento de trabalho $18 \mathrm{~cm}$ e ângulo de $30^{\circ}$, cânula de alto fluxo ${ }^{2}$ de $4 \mathrm{~mm}$, trocarte agudo, trocarte rombo e sonda. A fonte de luz fria ${ }^{3}$ com lâmpada de xenônio ${ }^{4}$ de $175 \mathrm{~W}$, cabo de fibra óptica ${ }^{5}$ de $3 \mathrm{~mm}$ de largura e $250 \mathrm{~cm}$ de comprimento, monitor colorido ${ }^{6}$, unidade de controle de câmera ${ }^{7}$, cabeça de câmera $^{8}$ e material cirúrgico de cirurgia geral. A documentação foi realizada através de vídeo impressora ${ }^{9}$.

\footnotetext{
${ }^{1}$ Hopkins ${ }^{\circledR}$ Forward Obliqúe Telescope $30^{\circ}$ - Karl Storz GmbH \& Co

${ }^{2}$ Arthroscope sheath - Karl Storz GmbH \& Co

${ }^{3}$ Cold Light Fountain Xenon Nova - Karl Storz GmbH \& Co

${ }^{4}$ Xenon Spare Lamp, 175W, 15V - Karl Storz GmbH \& Co

${ }^{5}$ Fluid Light Cables - Karl Storz GmbH \& Co

${ }^{6}$ Color Monitor - Karl Storz GmbH \& Co

${ }^{7}$ Telecam® Câmera Control Unit - Karl Storz GmbH \& Co

${ }^{8}$ Telecam® Câmera Head - Karl Storz GmbH \& Co

${ }^{9}$ Vídeo Printer - Sony
} 
Para a técnica artroscópica colocou-se o animal em decúbito lateral e com a articulação do ombro em posição neutra $\left(160^{\circ}\right)$, fez-se a identificação dos pontos anatômicos de referência: acrômio, tuberosidade maior e tendão do Mm. infraespinhoso. Com agulha 30x8 realizou-se artrocentese craniolateral entre o acrômio e a porção caudal da tuberosidade maior em direção caudomedial, promovendo-se a distenção da articulação pela injeção de solução fisiológica até que houvesse resistência no embolo da seringa. Para a confecção do portal artroscópio, incisão cutânea com lâmina de bisturi no 15,1 a 2 cm caudal e $1 \mathrm{~cm}$ distal ao acrômio, e utilização do trocarter (rombo ou agudo) com a cânula para adentrar à articulação. Concomitante o membro foi tracionado pela extremidade a fim de distender o espaço intra-articular. A irrigação foi feita pela própria cânula. A agulha usada inicialmente para a distenção da articulação permaneceu como via de drenagem. Para a confecção do portal instrumental, introduziu-se agulha calibre 30x8 craniolateral entre o acrômio e a porção caudal do tubérculo maior em direção caudomedial. A sua localização foi confirmada pelo artroscópio e, adjacente ao ponto de entrada da agulha fez-se incisão de pele, tecido celular subcutâneo e cápsula articular com lâmina de bisturi no 15 , sob guia artroscópico. Durante o procedimento cirúrgico foram realizados movimentos de flexão, extensão, rotação, abdução e adução da articulação a fim de maximizar a visibilidade da cavidade articular.

A cartilagem articular foi observada para a presença de condromalácea, fissura, eburnação e retalho livre; e a sinóvia, para hiperplasia.

Avaliou-se a ocorrência de infiltração de tecidos moles periarticulares, graduando subjetivamente como leve, moderado ou severo.

\section{Resultados}


O peso dos 15 animais estudados variou de 5 a $35 \mathrm{~kg}$ (média de 23,2kg).

\section{Estudo artroscópico}

Foram utilizados de 5 a 20ml de solução fisiológica para a distenção da cápsula articular, sendo a quantidade injetada diretamente proporcional ao porte do cadáver. $\mathrm{Na}$ falha da introdução correta da agulha no interior da cavidade articular houve acúmulo de líquido nos tecidos periarticulares, dificultando a identificação dos pontos anatômicos de referência e tornando cada vez mais difícil a sua distensão.

As estruturas tendão do Mm. bíceps braquial (Figura 1), ligamento glenoumeral medial e lateral, lábio glenóide, tendão do Mm. subescapular, bolsa articular caudal, cartilagem da superfície da cabeça do úmero cranial e caudal e da superfície da fossa glenóide foram observadas em todas as articulações nas quais se conseguiu a introdução do artroscópio.

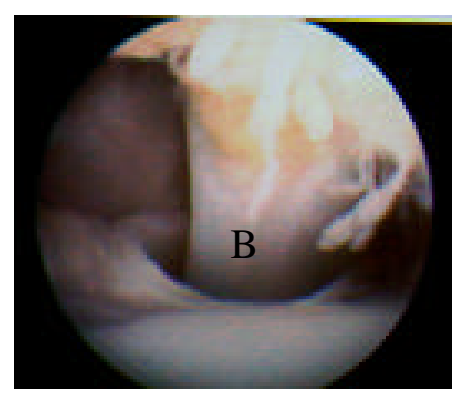

FIGURA 1 - Visão artroscópica do tendão do Mm. Bíceps braquial (B) na articulação do ombro em cadáver de cão.

A articulação estudada mostrou-se difícil para a confecção dos portais, seja artroscópico ou instrumental, o que foi sendo amenizado com o aprendizado. 
Em 15 (50\%) articulações estudadas a criação do portal artroscópico foi árdua, exigindo várias tentativas para a introdução da cânula e trocarte dentro da articulação; em 4 articulações não foi possível adentrar a articulação com o artroscópio e, portanto dar prosseguimento ao exame. A fim de facilitar este procedimento optou-se por realizar uma incisão cutânea maior do que a proposta inicialmente (cerca de 3 a $4 \mathrm{~cm}$ contra 1 a $2 \mathrm{~cm}$ ) e divulsionar o músculo deltóide, localizando-se o espaço articular por palpação digital.

A confecção do portal instrumental foi custosa em 19 (73,1\%) de 26 articulações (excluíram-se as articulações onde não foi possível a confecção do portal artroscópico já que este é essencial para a criação do portal instrumental). Ocorreu insucesso em 4 articulações.

Durante a inspeção da articulação ou realização da triangulação houve o deslocamento do artroscópio em 24 articulações (92,3\%). Em 1 articulação não foi possível o seu reposicionamento dentro da cavidade articular.

A triangulação foi difícil em 16 articulações (61,5\%). Em 4 articulações não foi possível localizar a agulha ou lâmina de bisturi com o artroscópio durante confecção do portal instrumental. Em 1 articulação a posição do portal instrumental ficou inadequada para a triangulação.

De um total de 22 articulações, em que o portal de instrumento foi completamente confeccionado, ocorreu remoção prematura do instrumental em 14 (63,6\%).

Ocorreu lesão iatrogênica de cartilagem articular em 17 (65,4\%) articulações durante a confecção do portal artroscópico; em 19 (73,1\%) para a construção do portal instrumental e em 16 (61,5\%) no momento da triangulação.

Em nenhuma das articulações estudadas houve laceração acidental de tendão. As lesões encontradas durante o exame artroscópico foram: condromalácea (8 
articulações - 30,8\%), eburnação (4 - 15,4\%) e fissura (4 - 15,4\%). Irregularidade no bordo lateral da cabeça do úmero foi visibilizado em uma articulação. Em uma articulação constatou-se a presença de material estranho fragmentado na bolsa articular caudal (Figura 2).

Detectou-se hiperplasia de sinóvia em 19 de 26 articulações (73,1 \%).

A infiltração dos tecidos moles periarticulares evidenciou-se em 25 de 30 articulações $(83,4 \%)$, sendo discreta em 8, moderada em 15 e severa em 2.

Em 4 articulações observou-se lesões localizadas em região caudo medial da cabeça do úmero, características de osteocondrose da cabeça umeral - 15,4\% (Figura 3).

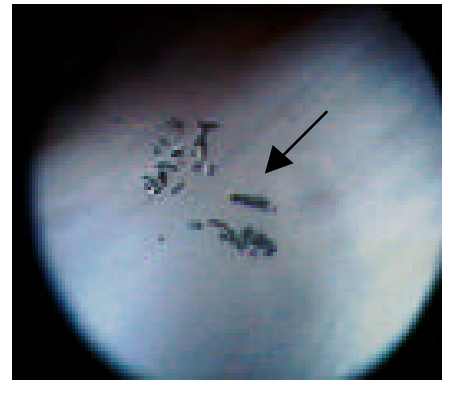

FIGURA 2 - Visão artroscópica de material estranho (seta) aderido a sinóvia em porção caudal da articulação do ombro em cadáver de cão.

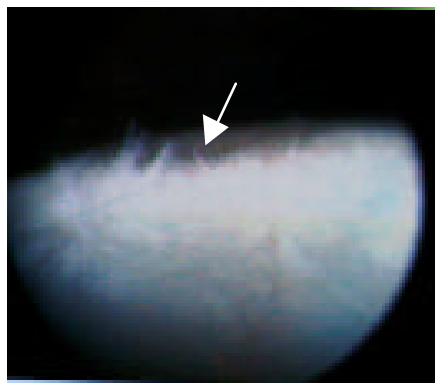

FIGURA 3 - Visão artroscópica de lesão de osteocondrose (seta) em porção caudo medial da cabeça do úmero na articulação do ombro em cadáver de cão.

\section{Estudo necroscópico}

Detectou-se lesão de cartilagem (escarificação leve a exposição do osso subcondral) consideradas lesão iatrogênica em 21 articulações estudadas. O local de maior prevalência da ocorrência desta lesão foi a superfície da cabeça do úmero cranial, porém também na porção caudal da cabeça do úmero e cavidade glenóide.

Quatro articulações apresentaram eburnação, condromalácea e fissura.

Hiperplasia de sinóvia foi notada em 12 articulações. 
Em duas articulações promoveu-se lesão iatrogênica na região lateral da cabeça do úmero próximo à inserção da cápsula articular, com perfuração de cartilagem e osso subcondral.

\section{Discussão}

A artroscopia é uma das técnicas endoscópicas de maior dificuldade no cão, requerendo intenso treinamento em cadáveres anterior a realização do exame em animais portadores de afecção. Sugere-se que o treinamento seja iniciado pela articulação do ombro $^{12}$, haja visto que é uma articulação favorável à artroscopia no cão devido ao seu tamanho e anatomia ${ }^{15}$.

Na espécie canina, a artroscopia possui uma curva de aprendizado lenta; inicialmente a identificação e distensão do espaço articular para a introdução da cânula e artroscópio, posteriormente orientação, exploração e identificação das estruturas intraarticulares e, finalmente, criação e visibilização de um portal instrumental através de triangulação ${ }^{10}$. Pôde-se vivenciar, neste estudo, os obstáculos do aprendizado da técnica artroscópica, sendo a manobra mais difícil a triangulação.

Segundo Siemering ${ }^{14}$ e Mc Carthy ${ }^{13}$ um exame completo não é passível de ser realizado em cães com menos de $6 \mathrm{~kg}$. Dentre as articulações estudadas, em 4 (dois cadáveres de 5 e $15 \mathrm{~kg}$ de peso) não foi possível a introdução do artroscópio, donde consideramos além do porte em um dos cadáveres a pouca experiência associada a dificuldade inerente da técnica.

Tomlinson $^{15}$ sugere uso do trocarte agudo para a perfuração da porção fibrosa da cápsula articular e rombo para a membrana sinovial; enquanto Rochat ${ }^{10}$ utiliza somente o trocarte rombo. Demos preferência a utilização do trocarte rombo, visto que promoveu 
menor lesão iatrogênica. A maior dificuldade consistiu em localizar o espaço articular e não na realização da perfuração da cápsula articular. $\mathrm{O}$ aumento da incisão facilitou este procedimento.

Na literatura consultada os autores estudados sugerem a técnica da triangulação para a confecção do portal instrumental, pela qual se dispõem o artroscópio e o instrumento em ângulos convergentes ${ }^{2,5,7,15}$. O nosso principal obstáculo para a criação do portal instrumental na posição protocolada foi localizar a agulha ou a lâmina de bisturi com o artroscópio, principalmente por ser uma região rica em vilos sinoviais, os quais não raro obstruíam a sua visibilização.

Dentre as dificuldades técnicas descritas citam-se o deslocamento quer do artroscópio ou do instrumental, requerendo seu reposicionamento ${ }^{1,12,13}$. Tal fato se deu principalmente durante a realização da triangulação e foi relativamente constante em nosso estudo, o que foi sendo amenizado com o treinamento.

A infiltração periarticular é uma complicação trans-operatória que pode interferir com o prosseguimento do exame artroscópico ${ }^{7,8,10,11,14}$. Quando da sua ocorrência, associada a massa muscular da articulação do ombro, a reintrodução do artroscópio ou instrumental após o seu deslocamento no orifício de origem nem sempre foi possível, havendo a necessidade da criação de novo portal e portanto agravando o extravasamento de líquido pela criação de novos orifícios. O uso de cânulas para a introdução de instrumentais no cão é contraditório entre os autores consultados; porém acreditamos que o seu uso nesta articulação, como preconizado por Mc Carthy ${ }^{13}$, minimizaria esta complicação.

A artroscopia, dotada de magnificação da imagem e iluminação, fornece um exame direto da cartilagem articular e membrana sinovial de visibilidade superior a artrotomia, ${ }^{3,4,6}$. Apesar do caráter apenas confirmativo de nosso estudo necroscópico, pôde-se notar a 
diferença de qualidade de avaliação das lesões de osteocondrose, presença dos fragmentos estranhos na bolsa caudal, lesão do ligamento glenoumeral medial e das próprias lesões iatrogênicas quando comparado ao estudo artroscópico.

Dentre as várias abordagens propostas para a articulação do ombro, tanto para o

portal artroscópico como instrumental ${ }^{1,9-11}$, a localização do portal artroscópico mostrou-se favorável para a visibilização das estruturas articulares propostas, enquanto o portal de instrumentos pareceu-nos mais favorável para a manipulação da região cranial da articulação, e talvez um portal instrumental caudal fosse mais adequado para o tratamento de lesões de osteocondrose, como preconizado por Beale et a $\mathrm{a}^{5}$.

\section{Conclusões}

$\mathrm{Na}$ articulação do ombro a maior dificuldade técnica foi a criação dos portais artroscópico e instrumental, este último sob triangulação. A identificação das estruturas intra-articulares, desde que o portal esteja bem posicionado é relativamente fácil.

O portal na localização descrita mostrou-se satisfatório para a visibilização das estruturas intra-articulares propostas.

A técnica requer treinamento intensivo e continuado.

\section{Referências}

1. Person MW. Arthroscopy of the canine shoulder joint. Comp Cont Ed Pract Vet 1986; 8(8): 537-48.

2. VanRyssen B, VanBree HJJ, Missinne S. Successful arthroscopic treatment of shoulder osteochondrosis in the dog. J Sm Anim Pract 1993; 34(10): 521-8.

3. VanBree HJJ, VanRyssen B. Diagnostic and surgical arthroscopy in osteochondrosis lesions. Vet Clin North Am (Sm Anim Pract) 1998; 28(1): 161-89.

4. Bardet JF. Traitement de l'ostéochondrite disséquante de l'épaule chez le chien par arthroscopie: étude rétrospective de 29 cas. Prat Med Chirur L'animal Comp 1995; 30(6): 685-94. 
5. Beale BS, Hulse DA, Schulz KS, Whitney WO. Small Animal Arthroscopy. Philadelphia: W. B. Saunders, 2003.

6. Wall CR, Taylor R. Arthroscopic biceps brachii tenotomy as a treatment for canine bicipital tenosynovitis. J Am Anim Hosp Assoc 2002; 38(2): 169-75.

7. Bardet JF. L' arthroscopie chez le chien et le chat. Bulletin de L' académie 2000; 73(2): 201-9.

8. Johnson AL, Hulse DA. Diseases of the joints. In: Fossum TW. Small Animal Surgery. 2ed. St. Louis: Mosby; 2002. p 1023-150.

9. Goring RL, Price C. Artroscopical examination of the canine scapulohumeral joint. J Am Anim Hosp Assoc 1987; 23(5): 551-5.

10. Rochat MC. Arthroscopy. Vet Clin North Am (Sm Anim Pract) 2001; 31(4): 76187.

11. Martini FM, Pinna S, Del Bue M. A simplified technique for diagnostic and surgical arthroscopy of the shoulder joint in the dog. J Sm Anim Pract 2002; 43(1): 7-11.

12. Abercromby R. Examination of arthroscopy as a clinical tool in small animal practice. J Sm Anim Pract 1997; 38(4): 174-8.

13. McCarthy TC. Arthroscopy. In: Freeman, L.J. Veterinary Endosurgery. St Louis: Mosby, 1999. p 237-50.

14. Siemering GH. Arthroscopy of dogs. JAVMA 1978; 172(5): 575-7.

15. Tomlinson J. Arthroscopy in dogs: Applications - SOTAL. In: 26th World Congress. 2001. Vancouver. World Sm Anim Vet Assoc Proc 2001; p 641-3.

Correspondência:

Angélica Cecília Tatarunas

Rua Victor Antonio Del Vecchio, 350/123

03124-070 São Paulo - SP

angelvet@usp.br

Data do recebimento: 08/02/2004

Data da revisão: 23/02/2004

Data da aprovação: 18/03/2004

Conflito de interesse: nenhum

Fonte de financiamento: FAPESP

\section{Como citar este artigo:}

Tatarunas AT, Matera JM. Estudo artroscópico da articulação do ombro em cadáveres de cães. Acta Cir Bras [serial online] 2004 Maio-Jun;19(3). Disponível em URL: http://www.scielo.br/acb. [also in CD-ROM]. 